REVISTA DE DERECHO UNED, NÚM. 18, 2016

\title{
EFICACIA, EFICIENCIA Y EFECTIVIDAD EN LA RESOLUCIÓN DE CONFLICTOS TRANSIGIBLES DE NIÑEZ Y ADOLESCENCIA EN EL ECUADOR
}

\author{
EFFICACY, EFFICIENCY AND EFFECTIVENESS OF \\ ALTERNATIVE DISPUTE RESOLUTION CASES FOR CHILDHOOD \\ AND ADOLESCENCE IN ECUADOR
}

Dra. Nathalia Viviana Lescano Galeas

Mayra Cristina Mena Mena

Abogada

DR. Rubén MÉndez Reátegui, PhD - DSc

Resumen: El presente artículo tiene como objetivo formular un análisis teórico de la resolución de conflictos transigibles de niñez y adolescencia a partir del caso ecuatoriano con énfasis en la provincia de Tungurahua. Se propone además la elaboración de un instrumento de evaluación preliminar (de carácter conceptual) a las vías previstas para la resolución de estos conflictos, denominado test de las tres $\mathrm{E}$, Eficacia, Eficiencia y Efectividad, el cual se enfoca en el acceso y provisión del servicio justicia desde el ámbito público y privado. No se introduce una crítica a las aproximaciones doctrinarias predominantes sino una propuesta que busca complementarlas. Por lo tanto, la investigación tiene un enfoque exploratorio y preliminar complementado a través de la técnica de entrevista, aplicada a mediadores y jueces, análisis de literatura especializada, normativa legal vigente, lineamientos de políticas públicas referente a la temática, datos estadísticos provenientes del Consejo de la Judicatura (vía judicial y mediación) y los obtenidos de los centros de mediación privados (vía mediación). Finalmente, se concluye que el sistema judicial está satu- 
rado y el Estado debe revisar su modelo de justicia para la resolución de conflictos vía mediación.

Abstract: This article aims a theoretical analysis of the of cases that can be settled out of court involving childhood and adolescence in Ecuador, with emphasis in the Tungurahua province. In addition, the paper posits the development of an instrument to assess alternative dispute resolution cases, named the Three $\mathrm{E}$ test. It refers to the concepts of Efficacy, Efficiency and Effectiveness focused on the provision of justice services from the public and private domains. The paper does not introduce a critique of the prevailing doctrinal approach instead seeks to enhance it, concluding that the judicial system is saturated and the Ecuadorian government must revise its model of justice for solving conflicts via mediation.

Palabras clave: eficacia, eficiencia, efectividad, conflicto transigible, mediación, niñez y adolescencia.

Keywords: Efficacy, efficiency, effectiveness, mediation, childhood and adolescence.

Recepción original: 18/02/2016

Aceptación original: 6/04/2016

Sumario: I. Introducción. II. Eficacia, eficiencia, y efectividad. II.1 ¿Cómo se entiende, eficacia, eficiencia y efectividad en el derecho? II.2 ¿En el acceso a la justicia es la mediación un método eficaz, eficiente y efectivo para la resolución de los conflictos transigibles en niñez y adolescencia? III. Las tres E, para la resolución de conflictos transigibles en niñez y adolescencia. IV. Conclusiones.

\section{INTRODUCCIÓN}

La Eficacia, Eficiencia, y la Efectividad (las tres E) constituyen pilares en el ámbito del derecho fundamentalmente cuando la discusión se enfoca en el acceso y provisión del servicio justicia. Tal como lo analiza CORNELIO LANDERO (2014) ${ }^{1}$, en la resolución de conflictos mediante mecanismos alternativos, como la mediación, (las tres E) ${ }^{2}$ previstas en el marco institucional, constituyen garantías a ser respetadas en

${ }^{1}$ CORNELIO LANDERO, E. Los Mecanismos Alternativos de Solución de Controversias como Derecho Humano, Revista Castellano-Manchega de Ciencias Sociales Universidad Juárez Autónoma de Tabasco, 2014, 17, pp. 81-95.

${ }^{2}$ Este test se deriva de la clásica relación normativa que involucra la existencia de tres elementos ampliamente estudiados en términos jurídicos: Eficiencia, Validez y Eficacia. 
procedimientos cuya disposición puede incidir en los derechos de las personas, así lo señalan, el artículo 8 de la Convención Americana sobre Derechos Humanos, que reglamenta las garantías judiciales y el artículo 14 del Pacto Internacional de Derechos Civiles y Políticos. ${ }^{3}$ Así mismo, como valores intrínsecos del sistema jurídico tanto la Eficacia, la Eficiencia y la Efectividad han sido ampliamente estudiados por la doctrina, al respecto encontramos aportes de CALSAMIGLIA BLANCAFORT $^{4}$, PAZ-ARES RODRÍGUEZ ${ }^{5}$, MERCURO y MEDEMA ${ }^{6}$ y ZYWICKI y STRINGHAM ${ }^{7}$. Sin embargo, presentarlas operativamente de manera relacionada a modo de mecanismo de evaluación (test) y aplicadas con el objetivo de tener un mejor panorama de la resolución de conflictos transigibles de niñez y adolescencia, representa una contribución novedosa, es decir, constituye un aporte que permitirá evaluar el desempeño de los juzgados y centros de mediación.

La evaluación de la justicia alternativa, específicamente de la mediación para obtener un diagnóstico del desempeño de la misma, y poder contrastarla con la del juicio permite que se conozcan errores y aciertos en el desarrollo del servicio justicia en relación a los mecanismos previstos para los conflictos transigibles de niñez y adolescencia, como un punto de partida para evaluaciones subsiguientes. Pues tal como lo sostiene las Naciones Unidas ${ }^{8}$, en las directrices que establece específicamente con respecto a la mediación eficaz, «(...) las iniciativas de mediación improvisadas y poco coordinadas aunque se pongan en marcha con la mejor de las intenciones, no contribuyen al objetivo de lograr una paz duradera»; para lo cual los procesos deben contar con un apoyo político, técnico y financiero firme. En relación con esto, MÁRQUEZ ALGARA ${ }^{9}$, realizó un trabajo de investigación, de evaluación de la mediación, en sede judicial, empleando para ello, lo que denominó como «criterios», Eficacia, Eficiencia y Efectividad. Aunque la metodología introducida fue similar a la del presente docu-

${ }^{3}$ CORTE INTERAMERICANA DE DERECHOS. HUMANOS. Sentencia Caso Baena Ricardo y otros, serie C. No. 72, párrafo 129, 2001.

${ }^{4}$ CALSAMIGLIA A., Eficiencia y Derecho, Doxa, 1987, 4, pp. 267- 287.

${ }^{5}$ PAZ-ARES RODRÍGUEZ, C. y VALENCIA, J. Principio de Eficiencia y Derecho Privado, Estudios del Derecho Mercantil en homenaje al Profesor Manuel Broseta Pont, 1995, III, pp. 28-43.

${ }^{6}$ MERCURO, N. y MEDERNA, S. (1998). «Economics and the Law», New Jersey, EUA.

${ }^{7}$ ZYWICKI, T. J. and STRINGHAM, E. P. Common Law and Economic Efficiency, Septiembre 8, 2010.

${ }^{8}$ ORGANIZACIÓN MUNDIAL DE LAS NACIONES UNIDAS. Directrices para una Mediación Eficaz, 2012.

${ }_{9}^{9}$ MÁRQUEZ ALGARA, M. Evaluación de la Justicia Alternativa, Editorial Porrúa, México, 2012. 
mento, MÁRQUEZ ALGARA ${ }^{10}$ no conceptualiza los criterios, aplica definiciones dadas por el derecho a la mediación ni centra su atención en los asuntos transigibles de niñez y adolescencia.

En la presente investigación a partir de un enfoque exploratorio, se pretende realizar un diagnóstico de la resolución de conflictos transigibles de niñez y adolescencia, empleando para ello el test de las tres E. Para esto se presenta el acceso a la justicia como un servicio, que debe resolver los conflictos de manera adecuada, oportuna, justa y eficaz, en función a las vías previstas para su abordaje. Estas vías desde el Estado se encuentran representadas por la judicatura y en el caso de la iniciativa privada por los centros de mediación adscritos a fundaciones y/o universidades. Aplica los métodos, dogmático-jurídico, analítico y sintético, complementándose éstos a través de las técnicas cualitativa-cuantitativa, documental a través de la revisión y análisis de literatura especializada, normativa legal vigente en el Ecuador, lineamientos de políticas públicas referente a la temática; de campo, la entrevista, aplicada a mediadores y jueces; datos estadísticos provenientes del Consejo de la Judicatura en torno a la Resolución de conflictos transigibles en niñez y adolescencia, en los años 2014 y 2015 (vía judicial y mediación) y los obtenidos de los centros de mediación privados (vía mediación). Todo esto con la finalidad de discutir la situación de la resolución de conflictos transigibles de niñez y adolescencia con énfasis en las políticas públicas adoptadas por el Estado Ecuatoriano en la generación de estrategias que permitan implementar políticas públicas en la resolución alternativas de conflictos transigibles de niñez y adolescencia.

En el primer capítulo, se efectúa un acercamiento Ius-económico con la finalidad de responder a la siguiente interrogante ¿Cómo se entiende Eficacia, Eficiencia y Efectividad en el derecho? En el segundo capítulo, centraremos la discusión en torno a la siguiente pregunta ¿En el acceso a la justicia es la mediación un método eficaz, eficiente y efectivo para la resolución de los conflictos transigibles en niñez y adolescencia? De esta manera, se presenta el mecanismo de evaluación denominado test de las tres E, que parte de las siguientes conceptualizaciones: 1) Para NORTH ${ }^{11}$ la Eficiencia es entendida como la capacidad de la regla formal para permitir la transferencia de derechos (bienes y servicios) a sus usos más valiosos. Implica la capacidad para lograr un fin empleando los mejores medios posibles. Es un elemento de carácter cualitativo que pueden ser expresados en términos

${ }^{10}$ Ibid, p. 20.

${ }^{11}$ NORTH, D. C. Institutions, Institutional Change and Economic Performance, Cambridge, University Press Cambridge, 1990. 
cuantitativos. 2) Por otra la parte, la Efectividad que en el presente trabajo hace referencia a que el derecho, también produce efectos específicos. En este sentido, se alude a la capacidad del derecho de lograr el efecto que se desea o se espera. Además se debe considerar que las normas son efectivas cuando puede comprobarse que los individuos a quienes se dirige la prescripción, desarrollan las conductas descritas en la norma. Este es un elemento estrechamente ligado al factor Validez, descrito por la Teoría General del Derecho a través del aporte de autores como KELSEN ${ }^{12}$ y HART ${ }^{13}$. 3) Finalmente la Eficacia, se conceptualiza como enfocada en los aspectos relacionados con la Legitimidad Social entendida como el reconocimiento de los individuos y que constituye un factor relacionado al cumplimiento de la norma por parte de los actores sociales, es decir, tiene que ver con un elemento cuantitativo en el sentido que involucra la cuantificación del logro de una meta como sostienen ELSTER ${ }^{14}$ y MÉNDEZ. ${ }^{15}$

Si el Estado mantiene, un monopolio legal, para la prestación del servicio justicia, que de hecho lo tiene en la vía ordinaria juzgados, al carecer de competencia, por definición no posee los incentivos para realizar un trabajo eficiente. En cambio, si la provisión del servicio de mediación se puede operar a través de agentes privados sin incurrir en prácticas monopólicas, la prestación del servicio por parte del Estado, se verá obligado a competir con otras para ser demandada y esto traerá un manejo más responsable. Es así que, desde el Estado, con miras a alcanzar mayor penetración en el mercado, se puede promover el pago del servicio a través de la introducción de un subsidio a la demanda (no a la oferta para eliminar incentivos perversos) y de carácter temporal. ${ }^{16}$ Más o menos como el sistema de «voucher» para la educación en los países de la Organización para la Cooperación y el Desarrollo Económico (OCDE).

Sin embargo, no hay mecanismo perfecto y solo un desarrollo de la forma de proveer técnicamente el servicio será el factor que incida en su viabilidad. Si la mediación no se puede proveer a un bajo costo, desde el ámbito privado, en el mediano plazo, se genera una barrera o restricción institucional para consolidar el sistema de justicia

${ }^{12}$ KELSEN, H. Introducción a la Teoría Pura del Derecho, México, Universidad Autónoma de México, 1956.

${ }^{13}$ HART, H. El Concepto del Derecho, México, Editora Nacional, 1980.

${ }^{14}$ ELSTER, J. El Cemento de la Sociedad, Barcelona, Editorial Ariel, 1991.

${ }^{15}$ MÉNDEZ, REATEGUI, R. El seguro de desempleo en el Perú: una retrospección preliminar a través de la Teoría Jurídica, la Economía Neo Institucional y el Austrian Law and Economics, Revista de Derecho UNED, 2014, No 14, pp. 375-422.

${ }^{16}$ Lo cual constituiría una ejemplo de política de "segundo mejor» (second best policy). 
y fortalecer el marco institucional tal como describe MÉNDEZ ${ }^{17}$. Eso puede arrojar una consecuencia nefasta y a la mediación ecuatoriana le puede pasar (en buena cuenta) lo mismo que a la conciliación extrajudicial en el Perú (que no ha terminado de despegar como propuesta para potenciar el sistema de justicia). Finalmente, al considerar que la matriz cultural tiene mucho que ver, si las creencias del ecuatoriano le llevan a entender que es el Estado quien tiene siempre que prestar el servicio «justicia» se genera un problema, para la provisión eficiente ya sea estatal, mixta o privada tal como sostiene MÉNDEZ ${ }^{18}$.

\section{EFICACIA, EFICIENCIA, Y EFECTIVIDAD}

Los términos Eficiencia, Eficacia, y Efectividad, conocidos como (las tres E) han sido aplicados en el ámbito de la teoría general por JEAMMAUD $^{19}$, en el derecho administrativo por autores como NORMAN-MAJOR ${ }^{20}$ y GARDAIS ORDANZA ${ }^{21}$; en el derecho laboral por POSNER $^{22}$ y GARCÍA-VILLALUENGA, BOLAÑOS, HIERRO, GARRIGÓS, DEL HOYO DORADO y MERINO ${ }^{23}$ y posteriormente trasladado al ámbito público para evaluar la gestión administrativa por FLAY, BIGLAN. BORUCH, GONZÁLEZ CASTRO, GOTTFREDSON, KELLAM, MOSCICKI, SHINKE, VALENTINE y $\mathrm{JI}^{24}$ y legislativa relacio-

${ }^{17}$ MÉNDEZ REÁTEGUI, R. An Introduction to Institutional Coordination as an Alternate model for Neo Institutional Economic Analysis, Procesos de MercadoRevista Europea de Economia Politica, 2013, Vol. X, 2, pp. 151-200.

${ }^{18}$ MÉNDEZ REATEGUI, R. Una Reflexión Jurídico-Económica e Institucional del Sistema de Justicia Civil en el Perú, Revista del Instituto Colombiano de Derecho Procesal, 2014, pp. 121-156.

${ }^{19}$ JEAMMAUD, A. En torno al problema de la efectividad del derecho, Revista de Critica Jurídica, 1985, 1, pp. 5-15.

${ }^{20}$ NORMAN, M. Social Equity in Public Administration?, Journal of Public Affairs Education, 2006, 17, pp. 233-252.

${ }^{21}$ GARDAIS ONDARZA, G. El control de legalidad y la eficiencia y eficacia como principios jurídicos fiscalizables, Revista de Derecho de la Universidad Católica de Valparaíso, 2002, pp. 323-341.

${ }^{22}$ POSNER, R. Eciency and the Ecacy of Title VII, University of Pennsylvania Law Review, 1987,136, pp. 513-522.

${ }^{23}$ GARCÍA VILALUENGA, L., BOLAÑOS CARTUJO, I., HIERRO REQUENA, M., GARRIGÓS TEMBLEQUE, S., DEL HOYO MARTÍNEZ, P., DORADO BARBÉ, A., y MERINO ORTIZ, C. La familia dialoga y llega a acuerdos: la mediación familiar. Resolución de conflictos, Madrid, Instituto Complutense de Mediación y Gestión de conflictos (UCM), 2010,

${ }^{24}$ FLAY BIGLAN, R., BORUCH ROBERT, F., GONZÁlES CASTRO, F., GOTTFREDSON, D., SHEPPARD, K., MOSCICKI, E., SCHINKE, S., Jeffrey C. Valentine, y Peter Ji Prevention Science, DOI: 10.1007/s11121-005-5553, 2005, pp. 202-225. 
nada a la generación de bienes y servicios a través del empleo del término "calidad substantiva» introducido por AITKEN" ${ }^{25}$.

Desde la definición más general, dada por la Real Academia Española ${ }^{26}$ los términos Eficacia y Efectividad, son sinónimos y definidos como la «capacidad de lograr el efecto que se desea o se espera» aceptándose al término Eficiencia como cuasi-sinónimo al establecer como que este hace referencia a la «capacidad de disponer de alguien o de algo para conseguir un efecto determinado». Además aunque se establece que los términos Eficacia, Eficiencia y Efectividad tienen en común el ejemplificar la noción de "hacer efecto», sin embargo, como establece GARNER ${ }^{27}$ en la actualidad se acepta sus divergencias conceptuales tanto a nivel jurídico general como en el diseño de políticas de gobierno y otros aspectos relacionados a la vida en sociedad. ${ }^{28} \mathrm{Al}$ respecto y para efectos del presente trabajo parte de la conceptualización de la Eficacia, como el cumplimiento de metas y objetivos o necesidades, a la Eficiencia como el cumplimiento de objetivos al menor costo posible y a la Efectividad como la diferencia entre objetivos planteados y resultados obtenidos, es decir, apunta a resolver de modo correcto.

\section{II.1 ¿Cómo se entiende, eficacia, eficiencia y efectividad en el derecho?}

En el ámbito jurídico, al hacer referencia a los términos de Eficacia, Eficiencia, y Efectividad (las tres E), debemos considerar que éstos, se aplican en relación a la norma jurídica. A partir del enfoque de la teoría general del derecho (respecto al proceso de creación de las mismas y su posterior aplicación); o desde el enfoque del derecho económico (en atención de las ideas de obediencia, aplicación de normas y cumplimiento de metas).

Además para este trabajo y a partir de la teoría general del derecho, el término Eficacia se encuentra estrechamente vinculado a la

${ }^{25}$ AITKEN, V. An exposition of legislative quality and its relevance for effective development, ProLaw Student Journal, 2013, Volume 2, pp. 1-43.

${ }^{26}$ REAL ACADEMIA DE LA LENGUA, Diccionario de la lengua española, Edición del Tricentenario, 2015.

${ }^{27}$ GARNER, A. B. Oxford Dictionary of Modern Legal Usage, Oxford University Press, 3 edition, 2001.

${ }^{28}$ BANCO INTERAMERICANO DE DESARROLLO, Eficacia, eficiencia, equidad y sostenibilidad: qué queremos decir? INDES Working paper series Publisher Departamento de Integración y Programas Regionales, Instituto Interamericano para el Desarrollo Social, 2001. 
teoría de las normas jurídicas. Desde la perspectiva de este autor se establece que la Eficacia, representa una condición de validez de las normas, es decir, que para ser válida una norma debe seguir el procedimiento establecido para su creación, lo que hace que se considere que dicha norma deba ser obedecida y aplicada, es decir validada por el tejido social.

Asimismo, la Eficacia de la norma en el derecho, será verificable en el momento que la misma sea puesta en práctica y por consiguiente obedecida por el «volk» (pueblo) y aplicada por los «kampfgericht» (jueces). Al respecto HUERTA OCHOA ${ }^{29}$, sostiene que, las normas jurídicas deben mantener una convergencia entre validez y eficacia y no únicamente limitarse a cumplir con los procedimientos establecidos para su formación sino prevalecer en la aplicación frente a otras normas jurídicas. En síntesis, la Eficacia implica que las normas deben adquirir fortaleza y reflejarse a través de su obligatoriedad y relacionándose a su vez a la «rechtsanwendung» (aplicación) y «rechtskonformität» (cumplimiento) de las normas previstas para su creación; pero de igual manera las normas elaboradas deben ser obedecidas, sin que para ello se tenga que recurrir a un juez, de no ser así la norma necesariamente deberá ser aplicada por el juez para que sea considerada como eficaz.

Estos términos no dejan de tener significados controvertidos y requieren que en parte se considere el debate existente con relación al "ambiguo y equívoco tema de la obediencia al Derecho» como establece HART $^{30}$ quien además describe a la Eficacia como un principio que supone que la organización y la función administrativa deben estar diseñadas y concebidas para garantizar la obtención de los objetivos, fines y metas propuestos y asignados por el propio ordenamiento jurídico, con lo que debe ser ligado a la planificación y a la evaluación o rendición de cuentas tal como sostiene JINESTA ${ }^{31}$.

De la Eficiencia se puede decir que a partir de su concepción Ius-económica implica, obtener los mejores resultados con el mayor ahorro de costos. Al respecto VAQUER ${ }^{32}$ señala que este criterio ha sido extensamente desarrollado por el derecho púbico y admi-

${ }^{29}$ HUERTA OCHOA, C. Teoría del Derecho. Cuestiones Relevantes, México, Instituto de Investigaciones Jurídicas, 2008

${ }^{30}$ HART, op. cit, p.49.

${ }^{31}$ JINESTA, E. Evaluación de resultados y rendición de cuentas en la Administración Pública. La reforma al artículo 11 de la Constitución Política. Revista Iustitia, 2000, pp. 166-167.

${ }^{32}$ VARQUER CABALLERÍA, M. La Eficacia y la Eficiencia del Derecho a la Vivienda en España, Madrid, Editorial Iustel, 2011. 
nistrativo con el objetivo de «valorar la racionalidad en la asignación de recursos e ingresos». Además, según MENENDEZ ${ }^{33}$ su manifestación trasciende lo referente al carácter operativo general de los poderes públicos y de la administración, pues se encuentra insertada en su momento constitutivo y como objetivo imprescindible, por consiguiente representando una realidad que debe por lo tanto ser asumida y no meramente presumida. En este sentido, la Eficiencia como criterio asumido se encuentra definida por la Constitución de la República del Ecuador del $2008^{34}$ cuando establece en su artículo 66, numeral 25 que: «el derecho a acceder a bienes y servicios públicos y privados de calidad, con Eficiencia, Eficacia y buen trato, así como a recibir información adecuada y veraz sobre su contenido y características». Es necesario precisar que la Eficiencia en el sistema jurídico ecuatoriano también suele ser entendida como un criterio de optimización (tal como es abordado por la doctrina en el derecho administrativo) que posee tal como señala PAREJO ALFONSO un «carácter relativo al relacionar los medios con los fines jurídicos.

Para el profesor austriaco JEAMMAUD, ambos conceptos aluden a la característica implícita en una norma jurídica según «la cual las situaciones y conductas concretas que pretende regular tiene una determinada relación $»^{35}$. A pesar de esta disquisición preliminar, debemos diferenciar entre Eficacia y Efectividad en el ámbito de lo jurídico. En línea con DIEZ-PICAZO Y PONCE DE LEÓN ${ }^{36}$, esto es así pues la Efectividad se enfoca en dos aspectos puntuales: a) El reconocimiento de una norma como lineamiento rector por parte de aquellos a quienes va dirigida (espontánea absorción por parte del tejido social); y b) Lo concerniente a su aplicación en el mundo real (donde el rol clave lo adquieren los operadores jurídicos como los jueces e incluso aquellos quienes dan vida al sistema alternativo de resolución de conflictos, tal es el caso de los árbitros, los mediadores y los conciliadores). Por lo tanto, como establece CAPELLA ${ }^{37}$ la efectividad debe asociarse con el éxito de la observancia de las reglas jurídicas a partir de sus fines y objetivos generales y específicos.

${ }^{33}$ MENÉNDEZ, A. La lucha por una Administración Eficiente, en Homenaje al profesor D. Juan Luis Iglesias Prada, 2011, Extraordinario, pp. 54-59.

${ }^{34}$ CONSTITUCIÓN DE LA REPÚBLICA DEL ECUADOR. Registro Oficial 449 de 20 de octubre de 2008.

35 JEAMMAUD, op. cit, p. 6.

${ }^{36}$ DÍEZ PICAZO, L. Experiencias Jurídicas y Teoría del Derecho, Tercera ed., Barcelona, Editorial Ariel S. A, 1999.

${ }^{37}$ CAPELLA, J. El derecho como lenguaje, Barcelona, Editorial Ariel, 1968. 


\section{II.2 ¿En el acceso a la justicia es la mediación un método eficaz, eficiente y efectivo para la resolución de los conflictos transigibles en niñez y adolescencia?}

\section{Del conflicto al acceso a la justicia}

Esta sección se dedica al estudio de las tres E, aplicadas a la resolución de conflictos transigibles en materia de niñez y adolescencia, en donde el acceso a la justicia depende del servicio (de juzgados o centros de mediación) provisto desde el ámbito, público, mixto y privado, para la resolución de conflictos.

Históricamente, según DOMÍNGUEZ BILBAO y GARCÍA DAU$\mathrm{DER}^{38}$ la idea de conflicto ha tendido una concepción negativa, de ahí que los seres humanos, en su gran mayoría durante mucho tiempo han entendido al conflicto como algo negativo que hay que evitar por sus consecuencias desastrosas y que el surgimiento del mismo implica su resolución sin que se haga mayor reflexión en la forma de lograrlo. Al respecto RITZER ${ }^{39}$ sostiene que se han desarrollado dos teorías: a) La teoría funcionalista o de consenso, que explica el cambio social, generado como producto de un acuerdo tácito de manera lenta y ordenada y b) La teoría del conflicto, que surge para cuestionar una sociedad supuestamente estable y consensual, generando cambios de manera rápida y desordena, como resultado de la lucha de grupos. Estas teorías se encontraban presentes entre los griegos antiguos, a) en la teoría del consenso con Platón, y posteriormente se encuentran a Santo Tomás de Aquino, Hobbes y Rousseau, b) en el caso de la teoría del conflicto Aristóteles como pensador del conflicto, San Agustín, Maquiavelo y Locke.

Posteriormente en la teoría del conflicto se han producido otros aportes, desarrollados en los últimos años, específicamente desde la década de los 60, encontrándose como antecedente de los mismos pensadores de izquierda como Karl Marx, cuando habla de la lucha de clases y el cambio como fuente de progreso de la humanidad. Este tipo de enfoques son criticados por la manera violenta de su aplicación práctica, lo que la ha llevado, según ZIMMERMANN ${ }^{40}$ al fracaso.

${ }^{38}$ DOMÍNGUEZ BILBAO, R. y GARCÍA DAUDER, S. Introducción a la Teoría del Conflicto en las Organizaciones, Madrid, Servicio de publicaciones Universidad Rey Juan Carlos, 2002.

${ }^{39}$ RITZER, G. Teoría Sociológica Contemporánea, Madrid, McGraw Hill, 1993.

${ }^{40}$ ZIMMERMANN, A. Marxismo, comunismo y derecho: cómo el marxismo llevó al desorden y al genocidio en la antigua Unión Soviética, Revista de Economía y Derecho, 2012, Vol. 9, 34, pp. 97-144. 
Asimismo, RITZER ${ }^{41}$ sostiene que de Marx a Wright, a más de la lucha de clases, se teoriza sobre otros tipos de conflictos entre los diferentes grupos que conforman la sociedad, de donde la imaginación sociológica, permite captar la historia y la biografía del individuo, y la relación de ambas en la sociedad, es decir entender el contexto. Así la teoría del conflicto es la superación de la que se considera no como una teoría crítica de la sociedad, sino una reacción al funcionalismo estructural.

Según POOLE ${ }^{42}$ la noción de justicia ha sido tratada desde la filosofía jurídica y política, fundamentalmente vista como una virtud moral, al respecto Ulpiano la concibió como, dar a cada uno lo suyo; sin embargo esta idea fue desarrollada siglos atrás por Aristóteles; posteriormente con Santo Tomás de Aquino, se difunde por la orbe cristiana. Para Platón, en cambio, constituyó la integridad moral y equilibrio entre las potencias del alma y simultáneamente el equilibrio social. Ockham, por su parte desarrollo el concepto de justicia como una cualidad propia del deudor, entonces la persona justa era aquella que pagaba sus deudas en tiempo y en forma. Más adelante, la orientación de la justicia se invierte, ya no va desde la persona hacia los demás, sino desde los otros hacia persona, ya no como una cualidad, sino como un principio organizativo de reparto de bienes y de protección de la autonomía de los sujetos. Hobbes, por su parte hace énfasis en la ley como medio para conseguir la justicia. Kant, la desarrolla como un ideal colectivo, de la convivencia pacífica entre personas libres; mientras que Kelsen cuestiona la idea de enjuiciar moralmente al derecho y considera pertinente el debate público y racional, sobre el cuál se sigue trabajando la concepción de justicia.

Frente al conflicto, el estado, como el encargado de administrar y procurar el orden y la convivencia, implantó el acceso a la justicia, para la resolución de controversias, aplicándose para ello, fundamentalmente la vía judicial. Sin embargo para este efecto, actualmente y ante la ineficacia del sistema ordinario implantado de manera exclusiva desde el Estado, según BUSTELO ELICABE-URRIOL ${ }^{43}$ surgen como respuesta los métodos alternativos de solución de conflictos, MARC; los cuales tienen varias denominaciones MARC, (Métodos Alternativos de Resolución de Conflictos), RAC (Resolución Alternativa de Controversias) o según sus siglas en inglés ADR (Alternative Dispute Resolution). A partir de la década de los 70, se inició un pro-

${ }^{41}$ RITZER, op. cit., p. 77.

42 POOLE, D., Filosofía del Derecho, Ávila, Ucav, 2009.

${ }^{43}$ BUSTELO ELICABE-URRIOL, D. La Mediación Claves para su Comprensión y Práctica, Madrid, Hara Press-Tritoma, 2009. 
ceso de revalorización, de estos métodos desde la National Conference on the Causes of Popular Dissatisfaction with the Administration of Justice, dictada por BURGER ${ }^{44}$, presidente de la Corte Suprema, sobre la preocupación de la Administración de Justicia, para que esta no llegué a verse desbordada por un notable aumento de la litigiosidad que afecte su Eficacia. A partir del año 2000, han surgido iniciativas dedicadas a fomentar la utilización de la mediación y otros métodos alternativos que favorezcan a la cultura de paz, como, la declaratoria del Año Internacional de Cultura de Paz, la Declaración y Programa de Acción de la Cultura de $\mathrm{Paz}^{45}$ y la promoción por los premios nóbel de paz Decenio Internacional de una cultura de paz y no violencia para los niños del mundo (2001-2010). ${ }^{46}$ Según MUÑOZ RUEDA y MOLINA ${ }^{47}$ las Naciones Unidas dentro de sus Objetivos de Desarrollo Sostenible (2015) $)^{48}$ puntualmente en el Objetivo 16 establece lo siguiente «Promover sociedades pacíficas e inclusivas para el desarrollo sostenible, para todos y crear instituciones eficaces, responsables e inclusivas a todos los niveles». En el Ecuador los Métodos Alternativos, han sido reconocidos desde la Constitución del año 1998 y se mantienen en la actual del 2008.

\section{LAS TRES E, PARA LA RESOLUCIÓN DE CONFLICTOS TRANSIGIBLES EN NIÑEZ Y ADOLESCENCIA}

\section{Eficacia}

PAREJO ALFONSO ${ }^{49}$ sostiene que «para servir a los intereses generales se debe utilizar los medios más idóneos o proporcionados para conseguir el fin previsto, lo que significa cumplir un precepto jurídico, que puede ser controlado jurisdiccionalmente, concretamente la Eficacia». Pues así, la Eficacia está en relación al reconocimiento por parte de los individuos de las vías previstas para la resolución de los conflic-

\footnotetext{
${ }^{44}$ WARREN, B. A Need for Systemic Anticipation, en The Pound Conference: Perspectives on Justice in the Future, 1976, Leo Levin y Russell R. Wheeler Eds.

${ }^{45}$ PROGRAMA NACIONAL DE MEDIACIÓN Y CULTURA DE PAZ, 2011. Recuperado de http://www.funcionjudicial.gob.ec/www/pdf/mediacion/LibroMediacion.pdf.

${ }^{46}$ ORGANIZACIÓN MUNDIAL DE LAS NACIONES UNIDAS. Directrices para una Mediación Eficaz, Nueva York, NU Press, 2012

${ }^{47}$ MUÑOZ, A. F., y MOLINA RUEDA, B. Una Cultura de Paz compleja y conflictiva. La búsqueda de equilibrios dinámicos. Revista Paz y Conflictos. Granada, 2009, 3, pp. 44-61.

${ }^{48}$ ORGANIZACIÓN MUNDIAL DE LAS NACIONES UNIDAS. Objetivos de Desarrollo Sostenible, Nueva York, NU Press, 2015.

${ }^{49}$ PAREJO. A. La eficacia como principio jurídico de la actuación de la Administración Pública, Revista de Documentación Administrativa, 1989, 218, pp. 16-65.
} 
tos, en el caso que nos ocupa, juicio o mediación. En este sentido los conflictos judicializados fácilmente duplican al número de casos presentados a la vía de mediación, es decir las personas prefieren ir a juicio.

En relación a lo anterior la norma suprema ecuatoriana, garantiza a sus habitantes como uno de los fines del Estado, el derecho a una cultura de paz. Al respecto MUÑOZ RUEDA Y MOLINA ${ }^{50}$, mencionan que la cultura de Paz es «una idea que puede ser entendida con cierta facilidad gracias a los imaginarios colectivos en los que se apoya y la necesidad de una cultura con capacidad de orientar e implementar un mundo más pacífico". Al respecto el artículo 190 de la Constitución de la República del Ecuador prevé el reconocimiento de la mediación, arbitraje y otros mecanismos de resolución de conflictos en materias que por su naturaleza se pueda transigir. Si bien el sistema procesal es un medio para la realización de la justicia. Las normas procesales consagran los principios de simplificación, uniformidad y eficacia. (Art. 169). Para el caso de los conflictos de niñez y adolescencia, la normativa jurídica ecuatoriana remite al Código Orgánico de la Niñez y Adolescencia, (2003) que, en concordancia con el Código Civil (2005), señalan la posibilidad de transigir aquellos asuntos, en los que las partes puedan disponer y siempre que no implique renuncia de derechos, es decir en conflictos por alimentos, tenencia y visitas. Con lo que las vías de gestión de estos conflictos son: los juzgados (desde lo público) o centros de mediación (desde lo público, privado o mixto) para lo cual hay que considerar además la Ley de Arbitraje y Mediación vigente desde el año $1997 .{ }^{51}$ Pues si bien, sistema de justicia es uno solo, la mediación constituye una estrategia complementaria al sistema denominado ordinario que puede ser considerado y promovido como parte integrante del sistema de justicia. En el ámbito familiar a través de un proceso no jurisdiccional, voluntario y confidencial se posibilita la comunicación entre las partes, para que traten de plasmar los intereses comunes en un acuerdo viable y estable que resulte satisfactorio para ambas, y atienda, también, a las necesidades del grupo familiar, especialmente las de menores y discapacitados. Esto con un facilitador denominado mediador, que es un tercero imparcial, neutral, capacitado idóneamente y sin ningún poder de decisión.

Respecto al desarrollo de las políticas del Programa de Restructuración de la Función Judicial, se propone la creación de una nueva

${ }^{50}$ MUÑOZ y MOLINA RUEDA, $O p$. cit., p. 45.

${ }^{51}$ LEY DE ARBITRAJE Y MEDIACIÓN. Registro Oficial 417 de 14-dic-2006. Última modificación: 09-mar-2009. Recuperado de http://www.desarrolloamazonico.gob.ec/ wpcontent/uploads/downloads/2014/05/LEY-DE-ARBITRAJE-Y-MEDIACION-act.pdf 
dirección de acceso a los servicios de justicia y dentro de estas la Subdirección de Centros de Mediación y Justicia de Paz. Se estableció tres estrategias, aplicadas desde el mes de agosto de 2013: estrategias de información, que consisten en la difusión de los principios de los mecanismos alternativos y cultura de paz a través de medios de comunicación; estrategias de posicionamiento, para ofrecer a través de actividades prácticas los beneficios y alcances de la mediación en los conflictos cotidianos en aras de evitar así su judicialización; y, estrategias de impacto, su propósito es ofrecer actividades, mensajes y propuestas que modifiquen los comportamientos de la ciudadanía en su forma de responder y resolver sus conflictos propios.

Durante el año 2013, se estableció un acuerdo interinstitucional reflejado en la mesa nacional de Mediación y Cultura de Paz, para la formación de 99 mediadores; realización de 15 jornadas de derivación durante 8 semanas, en 12 centros de 8 provincias; un total de 1929 casos derivados, 597 audiencias instaladas y 398 acuerdos logrados; se creó una Subdirección de Mediación y Justicia de Paz; se emitió nuevo Instructivo de Registro para Centros de Mediación públicos y privados de todo el país para lo cual se realizó la reestructuración de Centros de Mediación judiciales de todo el país, con el objetivo de ampliar la cobertura del servicio de mediación a nivel nacional ${ }^{52}$.

Dentro de los principales acuerdos interinstitucionales, encontramos a los siguientes: 1) El Ministerio de Educación asumió el compromiso de trabajar en el tema de semilleros de convivencia y cultura de paz en instituciones educativas; 2) La Secretaría del Buen Vivir busca fortalecer el compromiso de construir espacios de solución pacífica de conflictos en las comunidades como una opción de mejoramiento de la calidad de vida y el buen vivir; y 3) La Defensoría Pública y Defensoría del Pueblo desarrollará el mecanismo de la mediación en todos los espacios que correspondan a estas instituciones.

Con un marco jurídico pro mediación durante 18 años, en el Ecuador, se ha contado con el funcionamiento de centros de mediación, exclusivamente privados. Luego de la aprobación de la Constitución de la República en el año 2008, se inicia una serie de cambios para la transformación de la justicia en el país. Por lo que en concordancia con lo establecido constitucionalmente, al respecto de la gratuidad en el acceso a la justicia, encontramos al Programa de Restruc-

52 INSTRUCTIVO DE DERIVACIÓN DE CAUSAS A CENTROS DE MEDIACIÓN. Registro Oficial 139 de 01 de agosto de 2007 Recuperado de http://www.funcionjudicial.gob.ec/www/pdf/mediacion/Instructivo\%20para\%20la\%20Derivacion $\% 20 \mathrm{de} \% 20$ Causas\%20Judiciales.pdf. 
turación de la Función Judicial, Eje Modelo Administrativo (2011), que ha tratado de responder la inquietud en relación a los conflictos de niñez y adolescencia, a través de la división de la competencia en juzgados únicos, multi-competentes y unidades de Familia, Mujer, Niñez y Adolescencia. Respecto a los centros de mediación, dentro de los fundamentos del programa se señala que el servicio en relación a la cobertura territorial es deficiente, estos centros son mayormente privados, sus servicios están en función al interés de quienes los crean y no son gratuitos, por esto se los considera como no accesibles a la población y por lo tanto no cumplen con el principio de acceso a la justicia, establecido por la normativa ecuatoriana. Según los datos del Consejo Nacional de la Judicatura ${ }^{53}$, sobre centros de mediación, oficialmente existen 63 en funcionamiento, de los cuales, una vez analizada la información, podemos indicar que el $25.40 \%$, son centros públicos y el $74.60 \%$ son centros privados de los cuales el $25 \%$ funcionan en universidades. En la provincia de Tungurahua, se encuentran registrados tres centros de mediación, de los cuales dos se mantienen en funcionamiento: el Centro de la Función Judicial y el Centro de Arbitraje y Mediación de las Cámaras de Comercio e Industrias de Tungurahua. Al respecto VARGAS ${ }^{54}$ señala que, «la gratuidad de la justicia lleva a tramitar judicialmente casos cuyos costos superan sus beneficios probables».

Los siguientes datos describen la diferencia entre el número de casos que se conocieron en la vía judicial frente a los que se trataron en la vía de mediación. De donde se desprende que la vía ordinaria es elegida de manera predominante, aunque la mediación como un método alternativo tenga mayores ventajas en el ámbito familiar.

Vías de Resolución de Conflictos (2013-2015)

\begin{tabular}{|l|c|c|}
\hline \multicolumn{1}{|c|}{$\begin{array}{c}\text { Vías de resolución de conflictos } \\
\text { (tenencia, alimentos y visitas) }\end{array}$} & $\begin{array}{c}\text { Período } \\
\text { de tiempo }\end{array}$ & $\begin{array}{c}\text { Número } \\
\text { de procesos }\end{array}$ \\
\hline $\begin{array}{l}\text { Vía ordinaria } \\
\begin{array}{l}\text { Unidad de Familia Mujer Niñez y Ado- } \\
\text { lescencia. }\end{array}\end{array}$ & $\begin{array}{c}\text { De enero 2014 } \\
\text { a octubre 2015 }\end{array}$ & 3.535 \\
\hline
\end{tabular}

${ }^{53}$ CONSEJO DE LA JUDICATURA. Redición de cuentas Enero-Diciembre 2014. Recuperadode: http://www.funcionjudicialtungurahua.gob.ec/index.php?option=com wrapper\&view =wrapper\&Itemid=326.

${ }_{54}$ VARGAS VIANCOS, J. Eficiencia en la Justicia en Los Jueces y la Información, 2003, Centro de Estudios de Justicia de las Américas CEJA, pág. 68-92. 


\begin{tabular}{|l|c|c|}
\hline \multicolumn{1}{|c|}{$\begin{array}{c}\text { Vías de resolución de conflictos } \\
\text { (tenencia, alimentos y visitas) }\end{array}$} & $\begin{array}{c}\text { Período } \\
\text { de tiempo }\end{array}$ & $\begin{array}{c}\text { Número } \\
\text { de procesos }\end{array}$ \\
\hline $\begin{array}{l}\text { Vial alternativa. } \\
\text { Centro de Mediación Función Judicial. }\end{array}$ & $\begin{array}{c}\text { De octubre 2013 } \\
\text { a septiembre 2015 }\end{array}$ & 852 \\
\hline $\begin{array}{l}\text { Centro de Arbitraje y Mediación de las } \\
\text { Cámaras de Comercio e Industrias de } \\
\text { Tungurahua. }\end{array}$ & $\begin{array}{c}\text { De enero 2014 } \\
\text { a septiembre 2015 }\end{array}$ & 27 \\
\hline
\end{tabular}

Elaboración: Propia.

Fuente: Consejo de la Judicatura y Centros de Mediación.

Al hablar de derivación de los conflictos es importante considerar que, los casos han ingresado al sistema ordinario y de ahí se remiten a mediación para que sean gestionados, con lo que la situación en el Ecuador es la siguiente:

Causas derivadas a Mediación (2014)

\begin{tabular}{|l|c|}
\hline \multicolumn{2}{|c|}{ Causas derivadas a mediación } \\
\hline Número de causas derivadas a mediación (Tungurahua). & 336,00 \\
\hline Número de causas derivadas a mediación (nacional). & $10.143,00$ \\
\hline
\end{tabular}

Elaboración: Propia.

Fuente: Dirección Nacional de Estadística y Estudios Jurimétricos.

\section{Eficiencia}

Ante la demanda de una mayor agilidad y eficacia del sistema jurisdiccional por parte del justiciable, la resolución de los conflictos apuesta por la participación en la toma de decisiones de las personas directamente afectadas por la controversia, ante la valoración de las ventajas que reducen tiempo; aumentan la participación de los actores y con ello su responsabilidad para comprometerse y cumplir dichos pactos, esto posibilita relaciones posteriores entre ellas y favorece que las partes adquieran la capacidad de solucionar otros futuros conflictos del mismo modo. La mediación, se presenta entonces como un instrumento de paz social, también para las familias, que no sólo debe atender a la finalidad de aliviar la carga de los juzgados, sino responder, más bien, a un concepto amplio de hacer justicia desde y para sus protagonistas. 
Es así que el Programa de Restructuración de la Función Judicial (2013), ha implementado dentro de la justicia una serie de cambios estructurales en el servicio y acceso a la justicia.

Inversión por vías de Resolución: (2015)

\begin{tabular}{|l|c|c|c|}
\hline \multicolumn{1}{|c|}{$\begin{array}{c}\text { Vías de } \\
\text { resolución }\end{array}$} & Competencia & $\begin{array}{c}\text { Número de } \\
\text { Juzgados }\end{array}$ & $\begin{array}{c}\text { Número de } \\
\text { Jueces }\end{array}$ \\
\hline \multirow{2}{*}{ Ordinaria } & A) Juzgados únicos & 6 & 6 \\
\cline { 2 - 4 } & $\begin{array}{c}\text { B) Juzgados multicom- } \\
\text { petentes }\end{array}$ & 24 & 24 \\
\cline { 2 - 5 } & $\begin{array}{c}\text { C) Unidades de familia, } \\
\text { mujer, niñez y ado- } \\
\text { lescencia }\end{array}$ & 56 & 222 \\
\hline Total: & Centros de mediación & 179 & 252 \\
\hline Alternativa & \multicolumn{2}{|c|}{179} & 99 \\
\hline Total: & & 86 & 99 \\
\hline
\end{tabular}

Elaboración: Propia.

Fuente: Consejo de la Judicatura y Centros de Mediación

Para CLAVIJO ${ }^{55}$ el «analizar eficiencia implica conocer los «insumos» (estructura de los procesos judiciales) y los «resultados» (celeridad de la evacuación y sus posibles 'cuellos de botella').

Es así que respecto a la Eficiencia y al considerar las ventajas que posee la mediación tales como, rapidez, menor costo, cuidado de la relación por mencionar algunas, frente a las desventajas del juicio siendo algunas de estas, la congestión, el costo, el desgaste emocional y la corrupción, surge la pregunta ¿Por qué los centros de Mediación no llegan a tener la afluencia de los juzgados? Al respecto, al analizar el conflicto y sus diversas vías para gestionarlo FRANCISCOVIK INGUNZA y TORRES ANGULO ${ }^{56}$, llegan a concluir que la cultura del litigio en la que vivimos es predominante, aunque la mediación tenga mayores ventajas frente al juicio tiene menos demanda. En este senti-

${ }^{55}$ CLAVIJO, S. Costos y eficiencia de la rama judicial en Colombia políticas de choque operativo, Bogotá, Asociación Nacional de Instituciones Financieras -CEE, 2011.

${ }^{56}$ FRANCISCOVIK INGUNZA, B., y TORRES ANGULO. C. La eficiencia de los medios alternativos o adecuados de resolución de conflictos frente al sistema procesal civil, Revista de la Facultad de Derecho y Ciencia Política Ricardo de Palma, 2013, 2, pp. 231-260. 
do CLAVIJO ${ }^{57}$ establece que: «(...) la Rama Judicial ha disfrutado hasta la fecha de una doble condición. De una parte, el grueso de la población le reconoce a la Rama la 'majestad' que ha mostrado a la hora de enfrentar los grandes temas de Estado, a nivel de las Altas Cortes».

La Secretaria Nacional de Planificación y Desarrollo señala que: «Para un verdadero acceso a la justicia, es importante establecer un sistema caracterizado por su accesibilidad, oportunidad, independencia, honestidad, transparencia, imparcialidad y eficiencia ${ }^{58}$. El Estado debe atender la demanda de espacios de resolución de conflictos, ya sean estos provistos desde lo público o privado, de manera satisfactoria. Remitiéndonos de esta forma al Programa de Restructuración de la Función Judicial, Eje Modelo Administrativo (2011), en la inversión a nivel nacional se refleja la implementación para el caso de la vía judicial según la división por la competencia: a) seis Juzgados únicos (6 jueces); b) veinte y cuatro. Juzgados multi-competentes (24 jueces); c) cincuenta y seis unidades de Familia, Mujer, Niñez y Adolescencia (222 jueces). Para mediación se cuenta con ciento setenta y nueve Centros a nivel nacional. De los datos presentados, se desprende que, a nivel nacional en torno a los conflicto de niñez y adolescencia, se proyectó la creación de un total de ochenta y seis entre juzgados y unidades (conformadas por más de un juzgado) que en porcentaje representa apenas el 17,52\% del total nacional.

$\mathrm{Al}$ analizar la inversión, de recursos materiales y talentos humanos, hecho desde lo público, en relación a lo privado, que adicionalmente debe hacer rentable la prestación del servicio de resolución de conflictos, podemos pensar que estamos frente a una competencia desleal, que monopoliza la prestación del servicio, lo cual repercute en que, la competencia es un fenómeno económico cuya configuración presenta ciertas dificultades, en razón de la gran variedad de mecanismos y modus operandi que se presenta dentro del complejo mundo de las relaciones comerciales. Según BENAVENTE ${ }^{59}$ para ello es importante tener en cuenta que en todos los mercados existen infinidad de empresas con parámetros en los que se desenvuelven las relaciones económicas -precios, calidad, publicidad, innovaciones, tecnología, otros-.

Referente al término Eficiencia tanto los juzgados como centros de mediación están bajo la vigilancia y control del Consejo Nacional de la

${ }^{57}$ Ibid, p. 17.

${ }^{58}$ SECRETARÍA NACIONAL DE PLANIFICACIÓN Y DESARROLLO SEMPLADES. Plan Nacional del Buen Vivir, 2013, p. 200.

${ }^{59}$ BENAVENTE, H. Tutela Jurídica del Consumidor y la Competencia, Lima, Editorial San Marcos, 2012. 
Judicatura (ente estatal). Esto debido a que el acceso a la justicia es constitucionalmente, un derecho, administrado por el Estado, que debe ser proporcionado bajo principios establecidos, ante lo cual el test de la tres E, resulta fundamental. Así la Constitución ecuatoriana, establece que la Judicatura, deberá velar por la transparencia y eficiencia de la Función Judicial (Art. 181). En el caso de los Centros de Mediación, la Judicatura ${ }^{60}$, concede un número de registro de funcionamiento, previo al cumplimiento de requisitos, por lo que es necesario acreditar: a) Lista de mediadores; b) Reglamento; C) Código de Ética; d) Plan de ejecución; e) Tarifario a ser aprobado para el cobro de servicios; y f) La infraestructura necesaria para el funcionamiento.

Durante los años siguientes el proceso de creación de la infraestructura judicial ha sido lento, en el Ecuador en la provincia de Tungurahua por ejemplo al 2015, se ha entregado el complejo judicial. Mediante los siguientes datos se denota la profunda diferencia entre la asignación de recursos, desde quienes suministran el servicio, (Estado y centro de mediación privado) en el siguiente gráfico:

Inversión Provincia Tungurahua (2014)

\begin{tabular}{|l|r|}
\hline \multicolumn{1}{|c|}{ Entidad } & Presupuesto anual \\
\hline Corte Provincial de Justicia de Tungurahua. & $11,202,422.53$ USD \\
\hline $\begin{array}{l}\text { Centro de Arbitraje y Mediación de las Cáma- } \\
\text { ras de Comercio e Industrias de Tungurahua. }\end{array}$ & 22,000 USD \\
\hline Diferencia & $10,202,422.53$ USD \\
\hline
\end{tabular}

Elaboración: Propia.

Fuente: Consejo de la Judicatura y Centros de Mediación.

En la «Encuesta Nacional de Empleo, Desempleo y Subempleo, realizada en diciembre de 2014 por el Instituto Nacional de Estadística y Censos (INEC), se incluyó un módulo sobre percepción de los servicios de justicia en la ciudadanía». Instrumentos que se elaboran desde la percepción de diversos grupos etarios a partir de los 16 años en adelante, provenientes de 31.092 familias a nivel nacional, con una cobertura de 347.814 personas, derivadas de sus respuestas se desprenden porcentualmente, la aceptación del funcionamiento y servicio recibido. Los porcentajes de aceptación podrían no necesariamen-

${ }^{60}$ CONSEJO NACIONAL DE LA JUDICATURA. Mediación y Cultura de Paz 2015. Recuperado de http://www.funcionjudicial.gob.ec/index.php/es/component/content/ article/63mediacion-y-cultura-de-paz/409-informacion-centros-de-mediacion.html 
te estar en relación al servicio recibido como tal; si no a lo que el Estado proyecta, por lo que la población puede asociar mejoramiento de la justicia como un sinónimo de mayor inversión económica.

\section{Calificación del Funcionamiento del Consejo Nacional de la Judicatura (2015)}

\begin{tabular}{|l|c|}
\hline \multicolumn{1}{|c|}{ Funcionamiento del Consejo de la Judicatura } & Porcentaje \\
\hline Excelente calificación. & $9,5 \%$ \\
\hline Buena calificación. & $52 \%$ \\
\hline
\end{tabular}

Elaboración: Propia.

Fuente: Instituto Nacional de Estadística y Censos (INEC) módulo sobre percepción de los servicios de justicia en la ciudadanía.

Satisfacción de usuarios/as de las Unidades Judiciales en Ecuador (2015)

\begin{tabular}{|l|c|}
\hline $\begin{array}{c}\text { Personas que han acudido a las Unidades Judiciales } \\
\text { del país }\end{array}$ & Porcentaje \\
\hline Totalmente satisfechos. & $16,9 \%$ \\
\hline Muy satisfechos. & $40,2 \%$ \\
\hline Medianamente Satisfechos. & $18,8 \%$ \\
\hline
\end{tabular}

Elaboración: Propia.

Fuente: Instituto Nacional de Estadística y Censos (INEC) módulo sobre percepción de los servicios de justicia en la ciudadanía.

En este sentido y respecto a la satisfacción de usuarios de Centros de Mediación (dentro de la judicatura) se refleja que la encuesta realizada por el Instituto Ecuatoriano de Estadísticas y Censos INEC (2014), en cuanto a la infraestructura y funcionamiento de las unidades judiciales la ciudadanía en un 12,6\% calificó como excelente y el 51,5\% buena.

Satisfacción del Servicio Centros de Mediación Ecuador (2015)

\begin{tabular}{|l|c|}
\hline \multicolumn{1}{|c|}{ Funcionamiento de los centros de mediación } & Porcentaje \\
\hline Excelente. & $12,6 \%$ \\
\hline Buena. & $51,5 \%$ \\
\hline
\end{tabular}

Elaboración: Propia.

Fuente: Instituto Nacional de Estadística y Censos (INEC) módulo sobre percepción de los servicios de justicia en la ciudadanía. 
Así mismo indica que por cuestiones de cultura profesional, los abogados se resisten al cambio a salir de su zona de confort, tendientes a buscar en el pasado a través de la jurisprudencia, entonces una de las acciones más arriesgadas se produce cuando miramos hacia el lado, para verificar nuestros criterios interpretativos mediante el derecho comparado, sin embargo los profesionales del derecho no miramos hacia adelante, para lo que no hay norma, siendo esto una deficiencia, en donde la aplicación de los MARC, es vista como una pérdida de ingresos; según CLAVIJO ${ }^{61}$ : "Tal vez uno de los indicadores más útiles a nivel agregado sobre costoeficiencia en el área de justicia provenga de relacionar el gasto público del sector con el cúmulo de procesos pendientes de evacuación».

Por su parte los jueces que entienden la justicia como la repartición de sentencias, considerándolo un rol al que no quieren renunciar, pues no se deja de reconocer la labor de la judicatura en la resolución de conflictos muy delicados en donde las partes se han visto limitados, lo que se cuestiona al respecto es la generalización.

Con esta óptica el mismo CLAVIJO ${ }^{62}$ señala que «el ciudadano del común le reprocha a la Rama Judicial (en su conjunto), y con razón, su tardanza y falta de eficacia en el día-a-día; es una justicia que no le llega pronta al hombre común».

Desde la perspectiva de los justiciables, se hace hincapié en el mito respecto a la justicia de donde, se los justiciables asumen a priori que tienen la razón y que por consiguiente el juez al estar envestido de autoridad se las otorgará, esto unido al desconocimiento de la métodos alternos incide en la todavía marcada prevalencia de la vía judicial. Finalmente, señala que cualquier política que afronte los mejores medios para resolver los conflictos debe tomar en cuenta no solo el marco general sino la resistencia al cambio.

\section{Efectividad}

MUÑOZ y MOLINA RUEDA ${ }^{63}$ sostienen que, «Aspirar a gestionar la complejidad de la Cultura de Paz no es tarea fácil, son necesarios esfuerzos individuales e institucionales, académicos y científicos, solidarios y cooperativos".

\footnotetext{
${ }^{61}$ CLAVIJO, Op. cit, p. 47.

${ }^{62}$ Ibid, p. 15.

${ }^{63}$ MUÑOZ y MOLINA RUEDA, Op. cit, p. 49.
} 
En Ecuador, mientras en la vía ordinaria la tendencia es la creación de juzgados especializados en los centros de mediación, se tratan asuntos de niñez y adolescencia, civiles, inquilinato, indemnizaciones, mercantiles, tránsito, contratación pública y laboral (Cuando la mediación sea solicitada por el empleador). Pero como se estableció VARGAS asumen las labores en el VII Seminario de Gestión Judicial «La inversión en justicia», (2010), más allá de la inversión en infraestructura «es fundamental que quienes asumen las labores administrativas sean realmente gente con competencias en gestión y no con competencias en otras materias o en ninguna ${ }^{64}$. Respecto a esto cuando los conflictos a ser resueltos sean sobre niñez y adolescencia, según señala la norma suprema, se aplicará una administración de justicia especializada y los operadores de la misma deberán ser debidamente capacitados para aplicar los principios de la doctrina de protección integral (art. 3 núm. 3, 169, 175, 190). En la provincia del Chaco en Argentina, por ejemplo los mediadores deben tener una formación específica para mediar en situaciones tan complejas y trascendentales para la vida de las personas, pues se encuentran mucho más comprometidas que en aquellos asuntos meramente patrimoniales y más aún si se considera que la familia es protagonista de un rol fundamental en el desarrollo de las relaciones humanas, el amor mutuo, posibilitar la compañía, la atención de necesidades sociales básicas y propende la socialización. Por lo tanto, según GARAYO ${ }^{65}$ no solo se trata de un abordaje del conflicto de manera no violenta, implica mejoramiento de la calidad de vida de nuestros semejantes). En el Ecuador país no se requiere tener una especialidad para ser mediador en asuntos de familia, en la provincia de Tungurahua, no existen mediadores con formación específica en mediación familiar en ninguno de los dos centros que prestan el servicio (Centro de la Judicatura y Centro de las Cámaras de Comercio e Industrias de Tungurahua).

Conexo al costo del servicio, si el centro es público o privado en asuntos de niñez y adolescencia: tenencia, visitas y alimentos, por lo general el servicio es gratuito; se cobra en el ámbito privado dependiendo de la institución patrocinadora. Sin embargo si se considera que «no existe almuerzo gratis» una alternativa que implique un sub-

${ }^{64}$ VARGAS, E. La Inversión Pública en VII Seminario de Gestión Judicial-CEJA, Centro de Estudios de Justicia de las Américas-CEJA., 2010, p. 24.

${ }^{65}$ GARAYO, B. A. Acción Civil como Consecuencia de la Acción Penal, Revista El Acuerdo, 2013, 18, p. 91. 
sidio a la demanda a través de un mecanismo de vales (vouchers) probablemente podría reemplazar eficientemente a las iniciativas de subsidio a la oferta que han sido las predominantes en todos los sectores en el Ecuador.

Pese a todo lo mencionado anteriormente, según REDORTA ${ }^{66}$, después de 10 años de haber abandonado el litigio como único sistema de resolución de conflictos, con base a su experiencia en el ejercicio de la profesión y en relación a la crisis de los sistemas judiciales clásicos, manifiesta que, mientras los conflictos aumentan las personas relacionadas a la gestión de la resolución se sienten frustradas o insatisfechas; el conflicto es multidisciplinar sin embargo su abordaje se efectúa desde una única visión disciplinar, por lo que se resuelven conflictos desde la confrontación pero la inquietud es, ¿Realmente se los resuelve? Pues ir a la raíz del problema es más efectivo, es decir trabajar con las causas (mediación) y no desde las consecuencias tal como sucede en el juicio, entonces, se formula la pregunta ies realmente efectiva la resolución de conflictos desde la confrontación?; la duda crece si tomamos en cuenta además que, la tendencia es al creciente aumento de leyes y con ello las contradicciones de la justicia formal, el autor sugiere revisar los mecanismos de respuesta es decir, el modelo de justicia.

Tanto la Eficacia, la Eficiencia y la Efectividad constituirán lineamientos rectores para el servicio y bien común. Por lo que si no se obtiene el fin para el cual ha sido establecido el servicio público o se hace con medios inapropiados o desproporcionados al fin perseguido y que no sean idóneos jurídicamente para gestión eficaz, eficiente y efectiva, para el interés general, se violentan mandatos legales.

\section{CONCLUSIONES}

El derecho de acceso a la justicia concebido por la normativa vigente como un servicio público para la resolución de conflictos, puede ser proporcionado desde el ámbito público, privado o mixto y por lo tanto, requiere de la observancia de principios y la introducción de indicadores de gestión para lo cual el test de las tres E puede resultar enormemente importante al proveer lineamientos concep-

${ }^{66}$ REDORTA, J. Entorno de los Métodos Alternativos de Solución de Conflictos, Revista de Mediación, 2009, 2, 3., pp. 28-39. 
tuales que permitirían un mejor diálogo entre jurisconsultos y encargados de la gestión administrativa del sistema. En este sentido, autores como GUINART I SOLA ${ }^{67}$, defienden la necesidad de desarrollar indicadores que contemplen aspectos como la eficacia, la eficiencia y la efectividad y que contribuyan al adecuado cumplimiento de la responsabilidad y de la legalidad que caracterizan a la actuación pública directa e indirecta.

En las políticas públicas implementadas para el mejoramiento del acceso a la justicia en los conflictos de tenencia, alimentos y visitas, vía juzgados y centros de mediación, el estado ha optado por incrementar la inversión de infraestructura y en contratación de funcionarios, para unidades judiciales y centros de mediación públicos, lo que dificulta la competencia de los centros de mediación privados.

En el Ecuador, según se desprende el caso de la provincia de Tungurahua, donde la resolución de conflictos transigibles de niñez y adolescencia (tenencia, alimentos y visitas) por la vía judicial, haciende al 80,09\% del total de conflictos reportados; mientras que por la vía de mediación se resolvió el 19,91 \% de un total de 4414 casos tramitados. Es evidente que la mediación no constituye un mecanismo reconocido por las partes en conflicto, por lo que prefieren mayoritariamente acudir ante el juez, con lo que la norma que prevé la solución de conflictos transigibles en niñez y adolescencia, no se aplicada por parte de los justiciables, aun cuando la mediación tenga mayores ventajas frente al litigio sobre todo para el ámbito el derecho de familia, es decir, la resolución de conflictos vía mediación resulta no ser eficaz eficiente y efectivo.

El modelo de justicia implementado en el Ecuador en los últimos años, no ha logrado motivar el uso de la mediación por parte de los involucrados en el conflicto, para lo cual se requiere una cultura de paz lo cual se podría establecer un subsidio a la demanda, mediante un sistema que bien podría ser el de vales o vouchers para evitar la generación de un monopolio estatal del servicio, y de esta manera garantizar eficacia, eficiencia y efectividad en la resolución de conflictos transigibles de niñez y adolescencia.

${ }^{67}$ GUINART I SOLA, J. M. Indicadores de Gestión Pública, Revista de Administración Pública Nueva Época 110, 2004, 39, 1, pp. 315-336. 


\section{REFERENCIAS}

\section{1) Bibliografía}

AITKEN, V., An exposition of legislative quality and its relevance for effective development, ProLaw Student Journal, 2013, Volume 2, pags. 1-43.

BANCO INTERAMERICANO DE DESARROLLO. Eficacia, eficiencia, equidad y sostenibilidad: qué queremos decir? INDES Working paper series Publisher Departamento de Integración y Programas Regionales, Instituto Interamericano para el Desarrollo Social, 2001.

BENAVENTE, H., Tutela Jurídica del Consumidor y la Competencia, Lima, Editorial San Marcos, 2012.

BUSTELO ELICABE-URRIOL, D., La Mediación Claves para su Comprensión y Práctica, Madrid, Hara Press-Tritoma, 2009.

CALSAMIGLIA A., Eficiencia y Derecho, Doxa, 1987, 4, pp. 267-287.

CAPELLA, J., El derecho como lenguaje, Barcelona, Editorial Ariel, 1968.

CLAVIJO, S., Costos y eficiencia de la rama judicial en Colombia políticas de choque operativo, Bogotá, Asociación Nacional de Instituciones Financieras -CEE, 2011.

CORNELIO LANDERO, E., Los Mecanismos Alternativos de Solución de Controversias como Derecho Humano, Revista CastellanoManchega de Ciencias Sociales Universidad Juárez Autónoma de Tabasco, 2014, 17, pp. 81-95.

CORTE INTERAMERICANA DE DERECHOS. HUMANOS. Sentencia Caso Baena Ricardo y otros, serie C. n. ${ }^{\circ}$ 72, párrafo 129, 2001.

DÍEZ PICAZO, L., Experiencias Jurídicas y Teoría del Derecho, Tercera ed., Barcelona, editorial Ariel S. A, 1999.

DOMÍNGUEZ BILBAO, R. y GARCÍA DAUDER, S., Introducción a la Teoría del Conflicto en las Organizaciones, Madrid, Servicio de publicaciones Universidad Rey Juan Carlos, 2002.

ELSTER, J., El Cemento de la Sociedad, Barcelona, Editorial Ariel, 1991.

FLAY BIGLAN, R., BORUCH ROBERT, F., GONZÁLES CASTRO, F., GOTTFREDSON, D., SHEPPARD, K., MOSCICKI, E., SCHINKE, 
S., JEFFREY C. Valentine, y PETER Ji. Prevention Science, DOI: 10.1007/s11121-005-5553, 2005, págs. 202-225.

FRANCISCOVIK INGUNZA, B. y TORRES ANGULO. C., La eficiencia de los medios alternativos o adecuados de resolución de conflictos frente al sistema procesal civil, Revista de la Facultad de Derecho y Ciencia Política Ricardo de Palma, 2013, 2, págs. 231-260.

GARAYO, B. A., Acción Civil como Consecuencia de la Acción Penal, Revista El Acuerdo, 2013, 18, p. 91.

GARCÍA VILALUENGA, L., BOLAÑOS CARTUJO, I., HIERRO REQUENA, M., GARRIGÓS TEMBLEQUE, S., DEL HOYO MARTÍNEZ, P., DORADO BARBÉ, A. y MERINO ORTIZ, C., La familia dialoga y llega a acuerdos: la mediación familiar. Resolución de conflictos, Madrid, Instituto Complutense de Mediación y Gestión de conflictos (UCM), 2010.

GARDAIS ONDARZA, G., El control de legalidad y la eficiencia y eficacia como principios jurídicos fiscalizables, Revista de Derecho de la Universidad Católica de Valparaíso, 2002, págs. 323-341.

GARNER, A. B., Oxford Dictionary of Modern Legal Usage, Oxford University Press, 3 edition, 2001.

GUINART I SOLA, J. M. Indicadores de Gestión Pública, Revista de Administración Pública Nueva Época 110, 2004, 39, 1, págs. 315-336.

HART, H., El Concepto del Derecho, México, Editora Nacional, 1980.

HUERTA OCHOA, C., Teoría del Derecho. Cuestiones Relevantes, México, Instituto de Investigaciones Jurídicas, 2008.

JEAMMAUD, A., En torno al problema de la efectividad del derecho, Revista de Critica Jurídica, 1985, 1, págs. 5-15.

JINESTA, E. Evaluación de resultados y rendición de cuentas en la Administración Pública. La reforma al artículo 11 de la Constitución Política. Revista Iustitia, 2000, págs. 166-167.

KELSEN, H., Introducción a la Teoría Pura del Derecho, México, Universidad Autónoma de México, 1956.

MÁRQUEZ ALGARA, M., Evaluación de la Justicia Alternativa, Editorial Porrúa, México, 2012.

MÉNDEZ REÁTEGUI, R., El seguro de desempleo en el Perú: una retrospección preliminar a través de la Teoría Jurídica, la Econo- 
mía Neo Institucional y el Austrian Law and Economics, Revista de Derecho UNED, 2014, No 14, págs. 375-422.

- An Introduction to Institutional Coordination as an Alternate model for Neo Institutional Economic Analysis, Procesos de MercadoRevista Europea de Economia Politica, 2013, Vol. X, 2, pags. 151-200.

- Una Reflexión Jurídico-Económica e Institucional del Sistema de Justicia Civil en el Perú, Revista del Instituto Colombiano de Derecho Procesal, 2014, págs. 121-156.

MENÉNDEZ, A., La lucha por una Administración Eficiente, en Homenaje al profesor D. Juan Luis Iglesias Prada, 2011, Extraordinario, págs. 54-59.

MERCURO, N. y MEDERNA, S. (1998). «Economics and the Law», New Jersey, EUA.

MUÑOZ, A. F., y MOLINA RUEDA, B., Una Cultura de Paz compleja y conflictiva. La búsqueda de equilibrios dinámicos. Revista Paz y Conflictos. Granada, 2009, 3, págs. 44-61.

NORMAN, M., Social Equity in Public Administration?, Journal of Public Affairs Education, 2006, 17, págs. 233-252.

NORTH, D. C., Institutions, Institutional Change and Economic Performance, Cambridge, University Press Cambridge, 1990.

ORGANIZACIÓN MUNDIAL DE LAS NACIONES UNIDAS. Directrices para una Mediación Eficaz, Nueva York, NU Press, 2012.

- Objetivos de Desarrollo Sostenible, Nueva York, NU Press, 2015.

PAREJO. A., La eficacia como principio jurídico de la actuación de la Administración Pública, Revista de Documentación Administrativa, 1989, 218, págs. 16-65.

PAZ-ARES RODRÍGUEZ, C. y VALENCIA, J., Principio de Eficiencia y Derecho Privado, Estudios del Derecho Mercantil en homenaje al Profesor Manuel Broseta Pont, 1995, III, págs. 28-43.

POOLE, D., Filosofía del Derecho, Ávila, Ucav, 2009.

POSNER, R., Eciency and the Ecacy of Title VII, University of Pennsylvania Law Review, 1987,136, págs. 513-522 Real Academia De La Lengua, Diccionario de la lengua española, Edición del Tricentenario, 2015. 
REDORTA, J., Entorno de los Métodos Alternativos de Solución de

Conflictos, Revista de Mediación, 2009, 2, 3., pags. 28-39.

RITZER, G., Teoría Sociológica Contemporánea, Madrid, McGraw Hill, 1993.

SECRETARÍA NACIONAL DE PLANIFICACIÓN Y DESARROLLO SEMPLADES. Plan Nacional del Buen Vivir, 2013, p. 200.

VARGAS, E., La Inversión Pública en VII Seminario de Gestión Judicial-CEJA, Centro de Estudios de Justicia de las Américas-CEJA., 2010, p. 24.

VARGAS VIANCOS, J., Eficiencia en la Justicia en Los Jueces y la Información, 2003, Centro de Estudios de Justicia de las Américas CEJA, pág. 68-92.

VARQUER, CABALLERÍA M., La Eficacia y la Eficiencia del Derecho a la Vivienda en España, Madrid, Editorial Iustel, 2011.

WARREN, B. A., Need for Systemic Anticipation, en The Pound Conference: Perspectives on Justice in the Future, 1976, Leo Levin y Russell R. Wheeler Eds.

ZIMMERMANN, A., Marxismo, comunismo y derecho: cómo el marxismo llevó al desorden y al genocidio en la antigua Unión Soviética, Revista de Economía y Derecho, 2012, Vol. 9, 34, págs. 97-144.

ZYWICKI, T. J. and STRINGHAM, E. P., Common Law and Economic Efficiency, Septiembre 8, 2010.

\section{2) Base legal}

CONSTITUCIÓN DE LA REPÚBLICA DEL ECUADOR. Registro Oficial 449 de 20 de octubre de 2008.

INSTRUCTIVO DE DERIVACIÓN DE CAUSAS A CENTROS DE MEDIACIÓN. Registro Oficial 139 de 01 de agosto de 2007.

LEY DE ARBITRAJE Y MEDIACIÓN. Registro Oficial 417 de 14-dic-2006. Última modificación: 09-mar-2009. 


\section{3) Listado de websites}

CONSEJO NACIONAL DE LA JUDICATURA. Mediación y Cultura de Paz 2015. Recuperado de http://www.funcionjudicial.gob.ec/index. $\mathrm{php} / \mathrm{es} / \mathrm{component} / \mathrm{content/article/63mediacion-y-cultura-de-}$ paz/409-informacion-centros-de-mediacion.html

MEDIACIÓN Y CULTURA DE PAZ 2011. Recuperado de http://www. funcionjudicial.gob.ec/www/pdf/mediacion/LibroMediacion.pdf 
